\title{
Synthesis of a Möbius carbon nanobelt
}

Yasutomo Segawa, ${ }^{1,2,3,4, *}$ Tsugunori Watanabe, ${ }^{3}$ Kotono Yamanoue, ${ }^{3,5}$ Motonobu Kuwayama, ${ }^{3,5}$ Kosuke Watanabe, ${ }^{1,4}$ Jenny Pirillo, ${ }^{6}$ Yuh Hijikata, ${ }^{6}$ and Kenichiro Itami ${ }^{3,4,5, *}$

1 Institute for Molecular Science, Myodaiji, Okazaki, 444-8787, Japan

2 Department of Structural Molecular Science, SOKENDAI (The Graduate University for Advanced Studies), Myodaiji, Okazaki, 444-8787, Japan

3 JST, ERATO, Itami Molecular Nanocarbon Project, Nagoya University, Nagoya 4648602, Japan

4 Graduate School of Science, Nagoya University, Nagoya 464-8602, Japan

5 Institute of Transformative Bio-Molecules (WPI-ITbM) Nagoya University, Nagoya 464-8602, Japan

6 Institute for Chemical Reaction Design and Discovery (WPI-ICReDD), Hokkaido University, Sapporo, Hokkaido 001-0021, Japan

*Correspondence to: segawa@ims.ac.jp (Y.S.), itami@chem.nagoya-u.ac.jp (K.I.)

\begin{abstract}
:
New technologies for the creation of topological carbon nanostructures have significantly advanced synthetic organic chemistry and materials science. While simple molecular nanocarbons with a belt topology have been constructed recently, analogous carbon nanobelts with a twist, i.e., Möbius carbon nanobelts (MCNBs), have not yet been synthesized due to their high intrinsic strain. Herein, we report the synthesis, isolation, and characterization of a MCNB. Calculations of strain energies suggested that large MCNBs are synthetically accessible. Designing a macrocyclic precursor with an odd number of repeat units led to a successful rational synthetic route via Z-selective Wittig reactions and nickel-mediated intramolecular homocoupling reactions, which yielded $(25,25)$ MCNB over 14 steps. NMR and theoretical calculations revealed that the twist moiety of the Möbius band moves quickly around the MCNB molecule in solution. The topological chirality originating from the Möbius structure was confirmed experimentally using chiral HPLC separation and CD spectroscopy.
\end{abstract}




\section{Main Text:}

Organic chemists have striven to realize a wide variety of structural features on the molecular scale in nanocarbons. For example, nanocarbons with spherical, sheetlike, cylindrical, or other exotic structures are usually obtained as mixtures upon applying high energy to appropriate carbon sources ${ }^{1-4}$. In this context, molecular nanocarbon science, where such substructures are created in a precisely controlled fashion, has attracted substantial attention, given that this approach is fundamentally able to circumvent the problem of the formation of such nanocarbon mixtures ${ }^{5,6}$.

The history of the development of molecular nanocarbon science can be classified in terms of topology. Cycloparaphenylene (CPP), which was first proposed in the 1930s, is a ring-shaped molecular nanocarbon that represents a partial structure of carbon nanotubes (CNTs) ${ }^{7-10}$. Although CPPs were initially difficult to synthesize due to their high intrinsic strain energies, several synthetic methods, such as those reported since 2008 by Jasti, Itami, and Yamago ${ }^{11-13}$, have enabled the creation of molecular nanocarbons that exhibit nontrivial topologies, such as cages ${ }^{14-17}$, catenanes, and knots ${ }^{18,19}$. The next breakthrough in this research field was the synthesis of a carbon nanobelt (CNB), achieved by our group in $2017^{20}$. The fully fused, belt-shaped topology of CNBs creates two non-convertible faces, i.e., the inner and outer faces. Since then, the synthetic chemistry of CNBs and related belt-shaped arenes has been intensively investigated worldwide in the context of the bottom-up construction of CNT substructures ${ }^{21,22}$ as well as the creation of new functional molecular nanocarbon materials.

The CNB structural feature of irreducible inner and outer faces can be extended to aromatic molecules with the topology of a Möbius strip, which is the simplest example of a non-orientable surface (Fig. 1a). Möbius-type molecules are found in nature ${ }^{23}$ and have been of interest in synthetic organic chemistry, as demonstrated by the first successful preparation of a non-conjugated Möbius-type double-stranded molecule by Walba and co-workers in 1982 (Fig. 1b, left) ${ }^{24}$. Aromatic single-stranded molecules with Möbius aromaticity were realized by Harges and co-workers in $2003^{25}$, and Möbius aromaticity was further investigated by Grażyński ${ }^{26}$ and Osuka27,28. Despite several examples of single-stranded Möbius molecules, ${ }^{29-32}$ double-stranded 
aromatic molecules with Möbius topology still remain limited due to the difficulties associated with their synthesis. As shown in Fig. $1 \mathrm{~b}$, saturated linkers $\left(-\mathrm{CH}_{2} \mathrm{O}-\right)$ or chalcogen atom linkers (-S-) are necessary to reduce the strain caused by the Möbius topology (Fig. 1b, center and right) ${ }^{33,34}$. Even though Möbius-type CNBs have been theoretically proposed since the $1990 \mathrm{~s}^{34-37}$, a synthetic methodology to introduce Möbius topology to fully fused and fully conjugated all-sp² carbon structures still remains to be developed in molecular nanocarbon science.

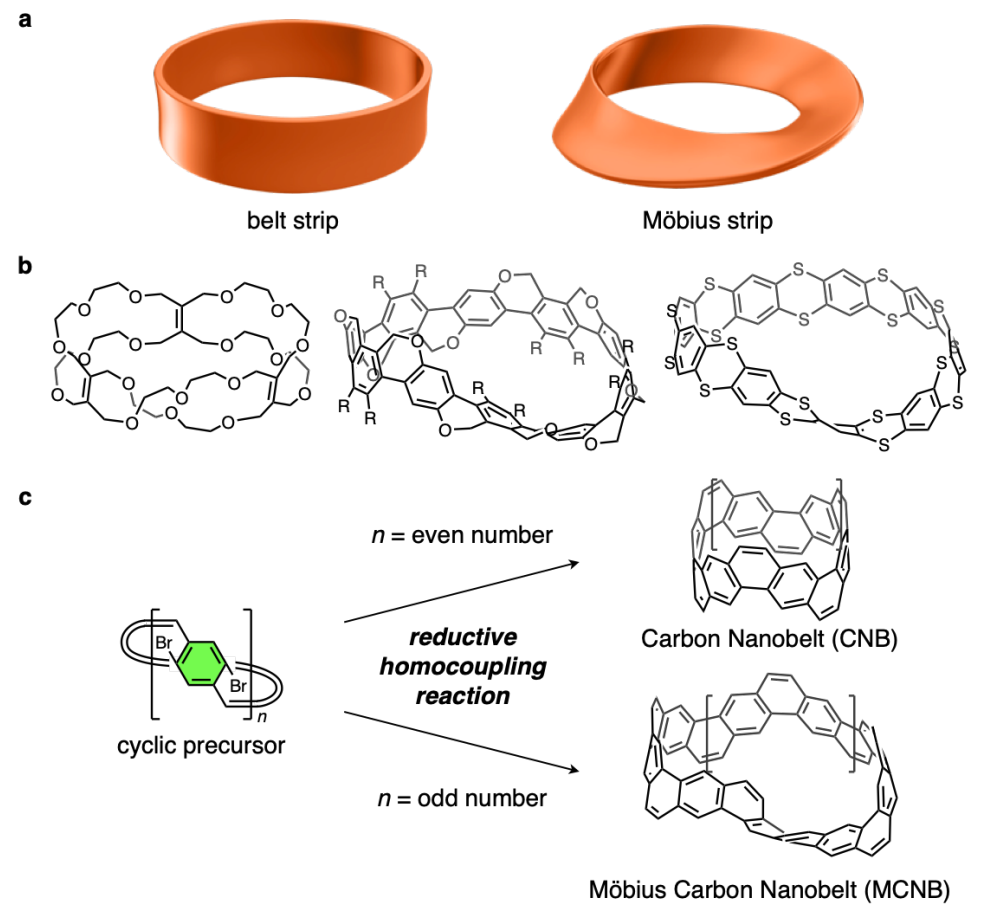

Fig. 1. Representative Möbius-strip molecules (a) Belt- (left) and Möbius-type (right) strips. (b) Belt-shaped double-stranded molecules with Möbius topology. (c) Carbon nanobelt (top right), Möbius carbon nanobelt (bottom right), and their respective synthetic strategies.

Herein, we report the synthesis, isolation, and optical analysis of a Möbius carbon nanobelt (MCNB), i.e., a fully fused carbon nanobelt with a twist. The key to the synthesis of such MCNBs is a modification of our previously reported synthetic strategy for $\mathrm{CNBs}^{19,38}$. As shown in Fig. 1c, $(n, n)$ CNBs $(n=6,8$, and 12, where $(n, n)$ is the chiral index of the corresponding CNTs) were synthesized via a reductive homocoupling reaction using cyclic molecules that consist of dibromoparaphenylene 
and cis-ethenylene precursors ${ }^{19,38}$. The important feature of this method is that a CNB can be generated when the number of repeat units is even, whereas an MCNB can be obtained when the number is odd. This is a simple but powerful method for the synthesis of a complex Möbius topology from highly symmetric precursors.

The target size of the MCNB was determined using DFT calculations. We found that MCNBs have a higher strain energy than CNBs with the same size (for details, see Fig. S1 in the Supplementary Information (SI)), and that the strain of the MCNBs is mainly induced during the final bond-formation step. Fig. 2a,b shows the hypothetical homodesmotic reactions using $(n, n) \mathrm{MCNBs},(n, n) \mathrm{CNBs}$, and their corresponding precursors ( $\operatorname{pre}(n, n) \mathrm{MCNBs}$ and pre $(n, n) \mathrm{CNBs}$ ), based on which the strain induced in the final bond-formation step $\left(\Delta H_{\mathrm{FBF}} / \mathrm{kcal} \cdot \mathrm{mol}^{-1}\right)$ was estimated. Cisstilbene (sti) and phenanthrene (phe) were used as reference molecules. For belts with a similar size, the $\Delta H_{\mathrm{FBF}}$ of the MCNB is much higher than that of the CNB (e.g., $\left.(6,6) \mathrm{CNB}: \Delta H_{\text {FBF }}=40.2 \mathrm{kcal} \cdot \mathrm{mol}^{-1} ;(7,7) \mathrm{MCNB}: 121.1 \mathrm{kcal} \cdot \mathrm{mol}^{-1}\right)$. As $(6,6) \mathrm{CNB}$ has been successfully synthesized using a nickel-mediated homocoupling reaction, the strain energy allowed by this synthetic method was estimated to be approximately 40 $\mathrm{kcal} \cdot \mathrm{mol}^{-1}$. Based on these considerations, the synthetic pathway and the symmetry of the product, $(15,15) \mathrm{MCNB}\left(\Delta H_{\mathrm{FBF}}=51.1 \mathrm{kcal} \cdot \mathrm{mol}^{-1}\right)$ and $(25,25) \mathrm{MCNB}\left(\Delta H_{\mathrm{FBF}}=\right.$ $\left.29.6 \mathrm{kcal} \cdot \mathrm{mol}^{-1}\right)$ were selected as the targets. The strain energies of the overall molecules are 85.7 and $49.4 \mathrm{kcal} \cdot \mathrm{mol}^{-1}$, respectively, indicating that the strain decreases with increasing size of the MCNB (for details, see Fig. S1 in SI). 

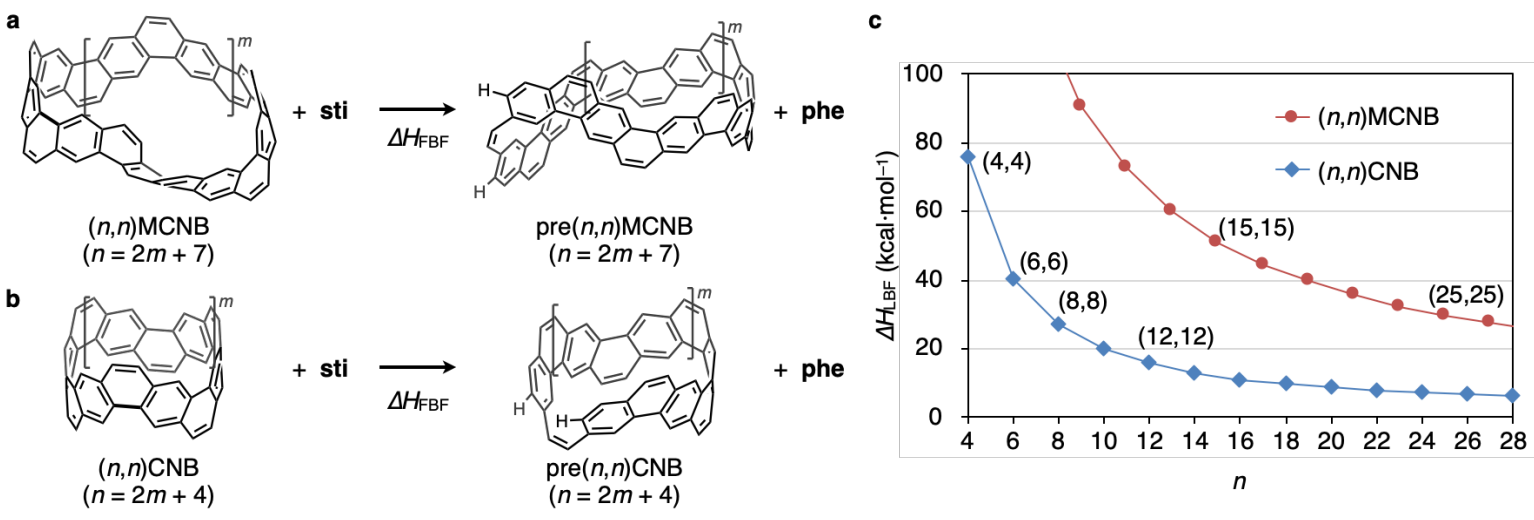

Fig. 2. Theoretical analysis of the intrinsic strain of MCNBs. $(\mathbf{a}, \mathbf{b})$ Hypothetical homodesmotic reactions to estimate the strain increase during the final $\mathrm{C}-\mathrm{C}$-bondformation steps. For pre(M)CNBs, bromine atoms are replaced with hydrogen atoms for clarity. Abbreviations: sti, cis-stilbene; phe, phenanthrene. (c) Plot of the heat of formation ( $\left.\triangle H_{\mathrm{FBF}}\right)$ for equations $\mathbf{a}$ and $\mathbf{b}$ as a function of the size of the MCNB.

Our synthetic route to MCNBs is shown in Fig. 3a,b. In order to improve the solubility of the intermediates and products, $n$-butoxy groups were introduced to the starting material 2 . Thus, $(25,25)$ MCNB with 20 butoxy groups (1) was targeted and synthesized from simple precursors 2 and $\mathbf{5}$ over 14 steps. First, the unsymmetric functionalization of phenanthrene $\mathbf{2}$ was investigated to ensure a Z-selective Wittig reaction sequence. During the screening of the Lewis-acid-catalyzed formylation of $\mathbf{2}$, we found that monoformylated 3 was obtained selectively using $\mathrm{TiCl}_{4}$ and $\mathrm{MeOCHCl}_{2}$ in high yield (75\%), and that a subsequent chloromethylation with $\mathrm{ZrCl}_{4}$ and $\mathrm{MeOCH}_{2} \mathrm{Cl}$ smoothly afforded bifunctional phenanthrene 4a with formyl and chloromethyl groups in $84 \%$ yield. The formyl and chloromethyl groups of $4 a$ were then converted into acetal and phosphonium groups, respectively, to yield $4 \mathbf{b}$. The sequential Wittig reaction of $\mathbf{5}$ with $\mathbf{4 a}$ followed by $\mathbf{4 b}$ produced key intermediate $\mathbf{7 c}$. Starting from $\mathbf{7 c}$ as the monomer, its dimer $(\mathbf{8 c})$, trimer $(\mathbf{9 c})$, and pentamer $(\mathbf{1 0 c})$ were synthesized via Wittig reactions. In these reactions, the formyl and phosphonium groups react selectively, since the chloromethyl and dimethylacetal groups were inert under the reaction conditions. The macrocyclization was performed with $\mathbf{1 0 d}$, which was derived from 10c and bears formyl and phosphonium groups to yield 11 in $67 \%$ yield. The reductive coupling of $\mathbf{1 1}$ with $\mathrm{Ni}(\mathrm{cod})_{2}$ and 4,4'-methoxycarbonyl-2,2'- 
bipyridyl gave $(\mathrm{BuO})_{20}(25,25) \mathrm{MCNB}$ (1) in $20 \%$ yield. In contrast, only trace mass peak corresponding to $(\mathrm{BuO})_{12}(15,15) \mathrm{MCNB}$ for the macrocycle 12 was observed under similar conditions (Fig. 3b, see Fig. S2 in SI for details). According to DFT calculations, the failure to generate the $(15,15) \mathrm{MCNB}$ structure might be due to the huge strain required for the formation of the final bond $\left(\Delta H_{\mathrm{FBF}}=51.1 \mathrm{kcal} \cdot \mathrm{mol}^{-1}\right)$.
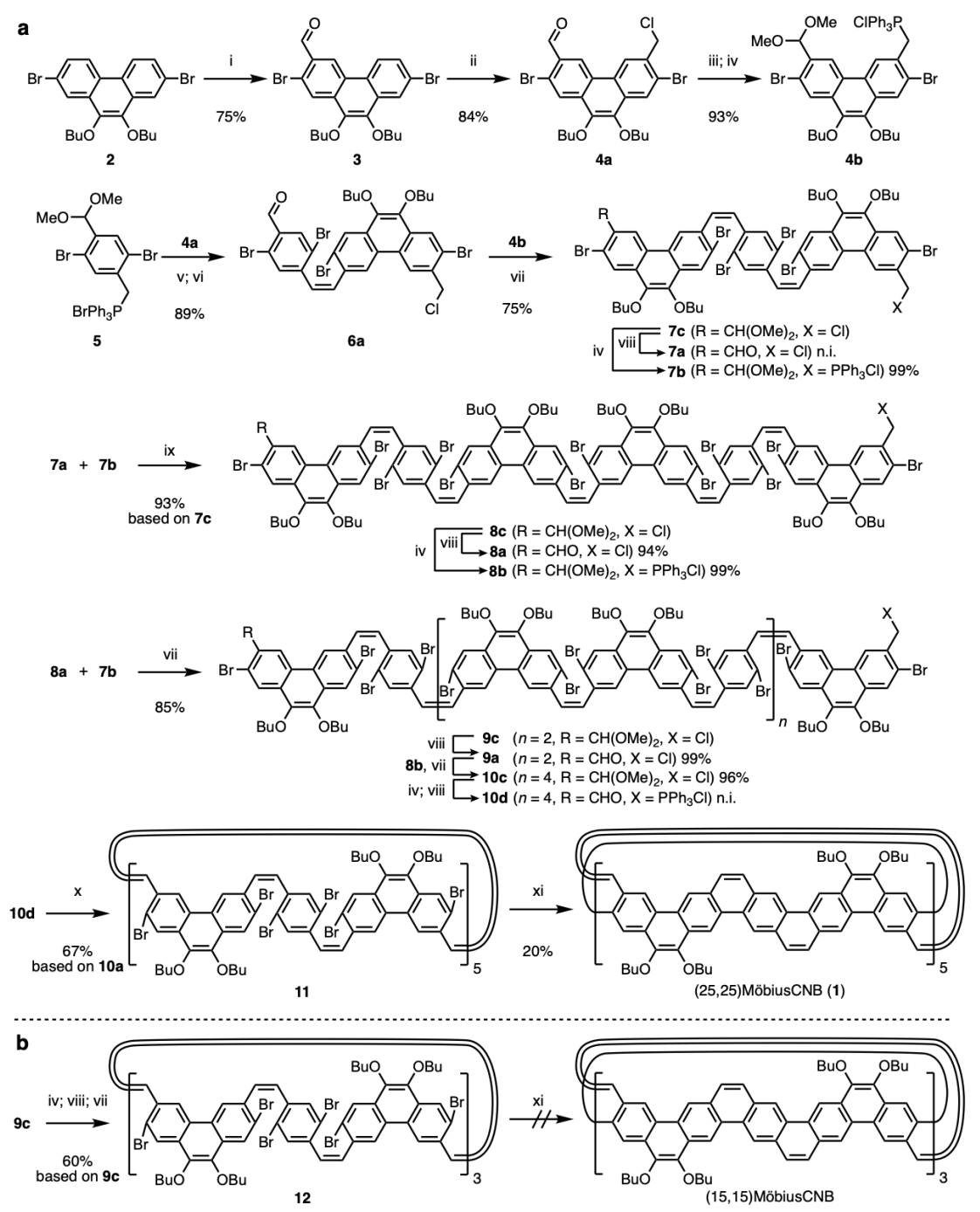

Fig. 3. Synthesis of a Möbius carbon nanobelt. (a) Synthetic route to $(25,25) \mathrm{MCNB}$; (b) Unsuccessful attempt to synthesize $(15,15) \mathrm{MCNB}$. Reagents and conditions: (i) $\mathrm{TiCl}_{4}, \mathrm{MeOCHCl}_{2}$, dichloromethane, $-45^{\circ} \mathrm{C}$; (ii) $\mathrm{ZrCl}_{4}, \mathrm{MeOCH}_{2} \mathrm{Cl}$, 1,2-dichloroethane, rt; (iii) $\mathrm{TsOH}, \mathrm{CH}(\mathrm{OMe})_{3}$, THF/methanol, rt; (iv) $\mathrm{PPh}_{3}, \mathrm{CH}(\mathrm{OMe})_{3}, 80-90{ }^{\circ} \mathrm{C}$; (v) $\mathrm{DBU}$, dichloromethane, $-10^{\circ} \mathrm{C}$; (vi) aq. $\mathrm{HCl}$, THF, $40^{\circ} \mathrm{C}$; (vii) TMG, THF, rt; (viii) TFA, chloroform, rt; (ix) LiHMDS, MS4A, chloroform/THF, $-78{ }^{\circ} \mathrm{C}$ to $-60{ }^{\circ} \mathrm{C}$; ( $\mathrm{x}$ ) $\operatorname{Pr}_{2} \mathrm{NEt}$, MS4A, chloroform, $0^{\circ} \mathrm{C}$; (xi) Ni(cod) 2, 4,4'-methoxycarbonyl-2,2'-bipyridyl, NMP, $70^{\circ} \mathrm{C}$. 
Abbreviations: Me, methyl; Bu, $n$-butyl; Ts, $p$-toluenesulfonyl; THF, tetrahydrofuran; DBU, 1,8-diazabicyclo[5.4.0]undec-7-ene; aq., aqueous solution; TMG, 1,1,3,3tetramethylguanidine; TFA, trifluoroacetic acid; HMDS, hexamethyldisilazide, MS, molecular sieves; cod, 1,8-cyclooctadiene; NMP, $\mathrm{N}$-methylpyrrolidone; rt, room temperature; n.i., not isolated.

The thus obtained Möbius belt $\mathbf{1}$ was characterized using high-resolution mass spectrometry (HRMS) and NMR spectroscopy. The high-resolution mass spectrum showed an isotope pattern with its highest peak at 3944.9449, which is in good agreement with the simulated pattern and mass number $(\mathrm{m} / \mathrm{z}=3944.9423)$ expected for $\mathrm{C}_{280} \mathrm{H}_{260} \mathrm{O}_{20}$ (for details, see Fig. $\mathrm{S} 3$ in $\mathrm{SI}$ ). The DFT-optimized structure of 1 shows $C_{2}$-symmetry with a long (38 $\AA$ ) and a short (30 $\AA$ ) axis (Fig. 4a). The broadened signals in the ${ }^{1} \mathrm{H}$ NMR spectrum observed at $25^{\circ} \mathrm{C}$ converged at $140^{\circ} \mathrm{C}$ to eight assignable singlet signals (Fig. 4b). These results indicate that the twist moiety of the Möbius belt moves quickly around the belt at high temperature. As shown in Fig. 4c, the molecular motion was simulated using density functional tight binding with molecular dynamics (DFTB-MD) calculation (for details, see the materials and method in SI). 


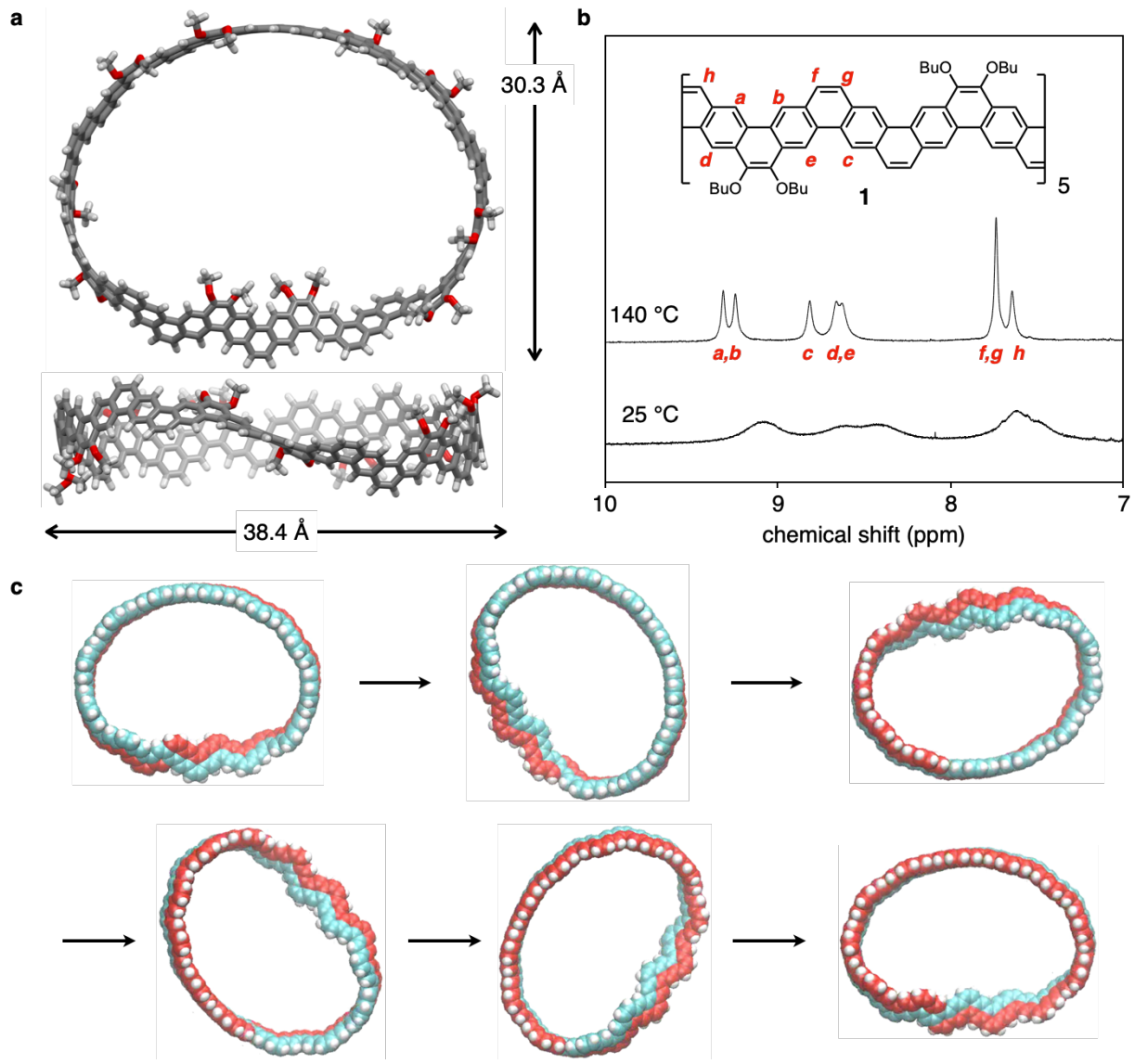

Fig. 4. Structure and dynamics of Möbius carbon nanobelt 1. (a) The structure of 1 was optimized at the B3LYP/6-31G(d) level of theory, whereby the butoxy groups were replaced by methoxy groups. (b) Aromatic region of the ${ }^{1} \mathrm{H}$ NMR spectra of 1 in 1,1,2,2-tetrachloroethane- $d_{2}$ at $25^{\circ} \mathrm{C}$ and $140{ }^{\circ} \mathrm{C}$. (c) Snapshots of the DFTB-MD simulation of $(25,25)$ MCNB (carbon: red or blue; hydrogen: white. For details, see Movie S1).

The photophysical properties of Möbius CNB 1 were also investigated. As shown in Fig. 5a, absorption maxima at 389 and $409 \mathrm{~nm}$ as well as a small absorption peak at $477 \mathrm{~nm}$ were observed, and greenish-blue fluorescence with maxima at 480, 513, and $551 \mathrm{~nm}$ were observed upon excitation at $380 \mathrm{~nm}$. Based on the fluorescence quantum yield (10\%) and half-life (14.1 ns), the radiative and nonradiative decay rate constants ( $k_{\mathrm{r}}$ and $k_{\mathrm{nr}}$ ) were estimated to be $7.1 \times 10^{6} \mathrm{~s}^{-1}$ and $6.4 \times 10^{7} \mathrm{~s}^{-1}$, respectively. Time-dependent density functional theory (TD-DFT) calculations of 1 suggested that unlike in the $D_{3 \mathrm{~h}}$-symmetric $(6,6) \mathrm{CNB}$, the $\mathrm{S}_{0} \rightarrow \mathrm{S}_{1}$ transition (assignable to the small band at $477 \mathrm{~nm}$ ) is symmetry-allowed $(f=0.6239)$, which reflects the lowered symmetry caused by the Möbius topology (see Fig. S4). The topological chirality of 1 
was also examined experimentally. Chiral separation of $\mathbf{1}$ was successfully achieved using chiral HPLC, and the circular dichroism (CD) spectrum of each fraction was collected (Fig. 5b and Fig. S5,6 in SI). Based on the CD spectra simulated using TDDFT calculations (for details, see Fig. $\mathrm{S} 6$ in $\mathrm{SI}$ ), the first and second fractions were tentatively assigned to $M$ - and $P$-chirality, respectively.
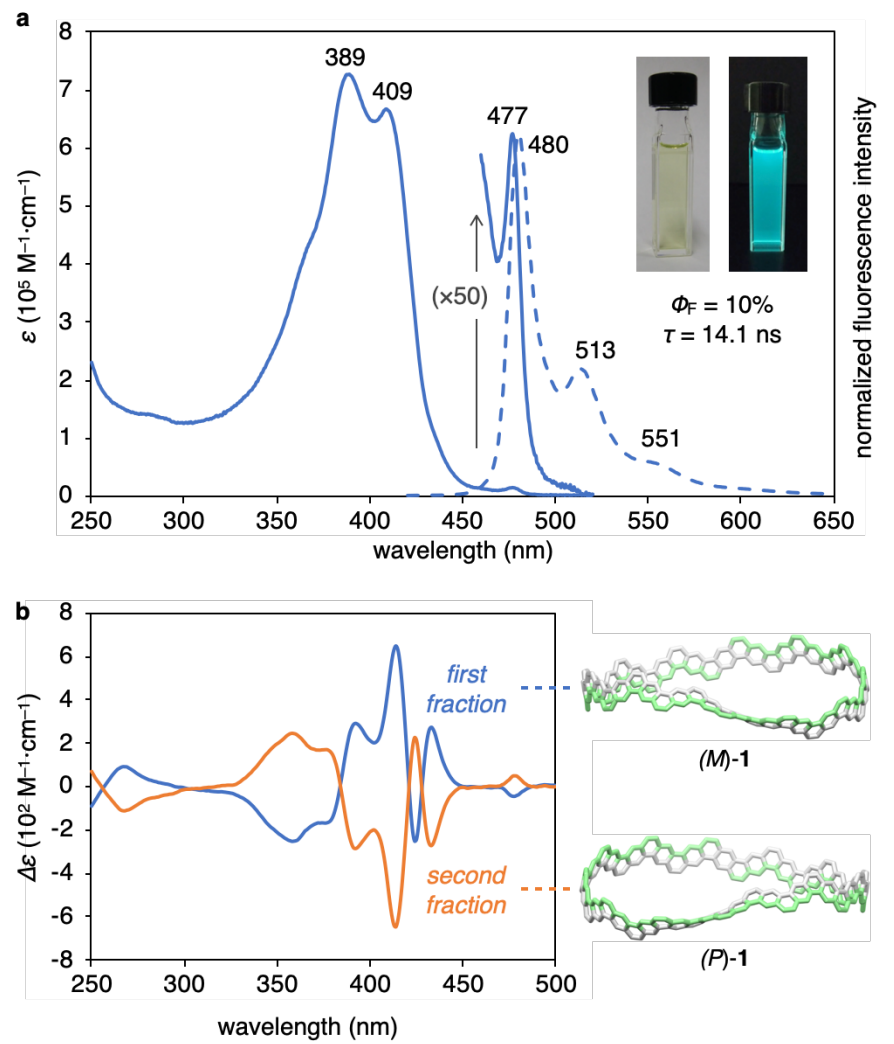

Fig. 5. Photophysical properties of Möbius carbon nanobelt 1. (a) Absorption and fluorescence spectra of a dichloromethane solution of 1. Insets show photographs of a dichloromethane solution of 1 with or without UV light irradiation (254 nm). (b) CD spectra of dichloromethane solutions of 1 separated using chiral column chromatography (CHIRALPAK-IE) with the tentative structural assignments ( $M$ or $P$ ); butoxy groups are omitted for clarity.

In conclusion, we have successfully synthesized a Möbius carbon nanobelt (MCNB), i.e., a topological molecular nanocarbon with a twist on armchair carbon nanobelts (CNBs). The strategy of using a variant of the previously used CNB 
precursor, cyclo(dibromoparaphenylene-Z-ethenylene), with an odd number of units led to the discovery of a rational synthetic route to such MCNBs. DFT calculations of the intrinsic strain energies suggested that the synthesis of MCNBs with large sizes would be most promising, and therefore, $(25,25) \mathrm{MCNB}$ was selected as the target. The synthesis was carried out via $Z$-selective Wittig and intramolecular homocoupling reactions with nickel complexes to yield decabutoxylated (25,25)MCNB (1) over 14 steps. NMR spectroscopy and DFTB-MD calculations revealed that the Möbius twist structure moves quickly around the molecule in solution. Photophysical measurements revealed that the synthesized MCNB exhibits greenish-blue fluorescence with a symmetry allowed $S_{0} \rightarrow S_{1}$ transition caused by the lowered symmetry. Experimentally, chiral HPLC separation and CD spectroscopy revealed that the chirality originates from the Möbius topology. The combination of strain calculations with a rational synthetic strategy can be expected to create a variety of topological molecular nanocarbons, which will promote the progress of materials science in this area. 


\section{References:}

1 Kroto, H. W., Heath, J. R., O'Brien, S. C., Curl, R. F. \& Smalley, R. E. C60: Buckminsterfullerene. Nature 318, 162-163 (1985).

2 lijima, S. Helical Microtubules of Graphitic Carbon. Nature 354, 56-58 (1991).

3 Novoselov, K. S. et al. Electric Field Effect in Atomically Thin Carbon Films. Science 306, 666-669 (2004).

4 Yang, F. et al. Chirality Pure Carbon Nanotubes: Growth, Sorting, and Characterization. Chem. Rev. 120, 2693-2758 (2020).

5 Segawa, Y., Ito, H. \& Itami, K. Structurally Uniform and Atomically Precise Carbon Nanostructures. Nat. Rev. Mater. 1, 15002 (2016).

6 Y. Segawa, D. R. Levine, K. Itami, Topologically Unique Molecular Nanocarbons. Acc. Chem. Res. 52, 2760-2767 (2019).

7 Parekh, V. C. \& Guha, P. C. Synthesis of pp'-Diphenylenedimonosulphide. J. Indian Chem. Soc. 11, 95-100 (1934).

8 Tahara, K. \& Tobe, Y. Molecular Loops and Belts. Chem. Rev. 106, 5274-5290 (2006).

9 Lewis, S. E. Cycloparaphenylenes and Related Nanohoops. Chem. Soc. Rev. 44, 2221-2304 (2015).

10 Segawa, Y., Yagi, A., Matsui, K. \& Itami, K. Design and Synthesis of Carbon Nanotube Segments. Angew. Chem. Int. Ed. 55, 5136-5158 (2016).

11 Jasti, R., Bhattacharjee, J., Neaton, J. B. \& Bertozzi, C. R. Synthesis, Characterization, and Theory of [9]-, [12]-, and [18]Cycloparaphenylene: Carbon Nanohoop Structures. J. Am. Chem. Soc. 130, 17646-17647 (2008).

12 Takaba, H., Omachi, H., Yamamoto, Y., Bouffard, J. \& Itami, K. Selective Synthesis of [12]Cycloparaphenylene. Angew. Chem. Int. Ed. 48, 6112-6116 (2009). 
13 Yamago, S., Watanabe, Y. \& Iwamoto, T. Synthesis of [8]Cycloparaphenylene from a Square-Shaped Tetranuclear Platinum Complex. Angew. Chem. Int. Ed. 49, 757-759 (2010).

14 Matsui, K., Segawa, Y., Namikawa, T., Kamada, K. \& Itami, K. Synthesis and Properties of All-Benzene Carbon Nanocages: A Junction Unit of Branched Carbon Nanotubes. Chem. Sci. 4, 84-88 (2013).

15 Kayahara, E. et al. Synthesis and Physical Properties of a Ball-like ThreeDimensional r-Conjugated Molecule. Nat. Commun. 4, 2694 (2013).

16 Matsui, K., Segawa, Y. \& Itami, K. All-Benzene Carbon Nanocages: Size-Selective Synthesis, Photophysical Properties, and Crystal Structure. J. Am. Chem. Soc. 136, 16452-16458 (2014).

17 Hayase, N., Nogami, J., Shibata, Y. \& Tanaka, K. Synthesis of a Strained Spherical Carbon Nanocage by Regioselective Alkyne Cyclotrimerization. Angew. Chem. Int. Ed. 58, 9439-9442 (2019).

18 Segawa, Y. et al. Topological Molecular Nanocarbons: All-Benzene Catenane and Trefoil Knot. Science 365, 272-276 (2019).

19 Segawa, Y., Kuwayama, M. \& Itami, K. Synthesis and Structure of [9]Cycloparaphenylene Catenane: An All-Benzene Catenane Consisting of Small Rings. Org. Lett. 22, 1067-1070 (2020).

20 Povie, G., Segawa, Y., Nishihara, T., Miyauchi, Y. \& Itami, K. Synthesis of a Carbon Nanobelt. Science 356, 172-175 (2017).

21 Cheung, K. Y., Segawa, Y. \& Itami, K. Synthetic Strategies of Carbon Nanobelts and Related Belt-Shaped Polycyclic Aromatic Hydrocarbons. Chem. Eur. J. 26, 14791-14801 (2020).

22 Guo, Q.-H., Qiu, Y., Wang, M.-X. \& Stoddart, J. F. Aromatic Hydrocarbon Belts. Nat. Chem. 13, 402-419 (2021).

23 Craik, D. J., Daly, N. L., Bond, T. \& Waine, C. Plant Cyclotides: A Unique Family of Cyclic and Knotted Proteins That Defines the Cyclic Cystine Knot Structural Motif. J. Mol. Biol. 294, 1327-1336 (1999). 
24 Walba, D. M., Richards, R. M. \& Haltiwanger, R. C. Total Synthesis of the First Molecular Moebius Strip. J. Am. Chem. Soc. 104, 3219-3221 (1982).

25 Ajami, D., Oeckler, O., Simon, A. \& Herges, R. Synthesis of a Möbius Aromatic Hydrocarbon. Nature 426, 819-821 (2003).

26 Stępień, M., Latos-Grażyński, L., Sprutta, N., Chwalisz, P. \& Szterenberg, L. Expanded Porphyrin with a Split Personality: A Hückel-Möbius Aromaticity Switch. Angew. Chem. Int. Ed. 46, 7869-7873 (2007).

27 Sankar, J. et al. Unambiguous Identification of Möbius Aromaticity for Meso-ArylSubstituted [28]Hexaphyrins(1.1.1.1.1.1). J. Am. Chem. Soc. 130, 13568-13579 (2008).

28 Yoon, Z. S., Osuka, A. \& Kim, D. Möbius Aromaticity and Antiaromaticity in Expanded Porphyrins. Nat. Chem. 1, 113-122 (2009).

29 Schaller, G. R. et al. Design and Synthesis of the First Triply Twisted Möbius Annulene. Nat. Chem. 6, 608-613 (2014).

30 Naulet, G. et al. Cyclic Tris-[5]Helicenes with Single and Triple Twisted Möbius Topologies and Möbius Aromaticity. Chem. Sci. 9, 8930-8936 (2018).

31 Fan, Y.-Y. et al. An Isolable Catenane Consisting of Two Möbius Conjugated Nanohoops. Nat. Commun. 9, 3037 (2018).

32 Jiang, $X$. et al. Kinetic Control in the Synthesis of a Möbius Tris((Ethynyl)[5]Helicene) Macrocycle Using Alkyne Metathesis. J. Am. Chem. Soc. 142, 6493-6498 (2020).

33 Nishigaki, S. et al. Synthesis of Belt- and Möbius-Shaped Cycloparaphenylenes by Rhodium-Catalyzed Alkyne Cyclotrimerization. J. Am. Chem. Soc. 141, 1495514960 (2019).

34 Wang, S. et al. Sulphur-Embedded Hydrocarbon Belts: Synthesis, Structure and Redox Chemistry of Cyclothianthrenes. Angew. Chem. Int. Ed. 60, 18443-18447 (2021).

35 Herges, R. Topology in Chemistry: Designing Möbius Molecules. Chem. Rev. 106, 4820-4842 (2006). 
36 Türker, L. MNDO Treatment of the Huckel and Mobius Types of Cyclacenes. J. Mol. Struct. THEOCHEM 454, 83-86 (1998).

37 Türker, L. \& Gümüş, S. Cyclacenes. J. Mol. Struct. THEOCHEM 685, 1-33 (2004).

38 Povie, G., Segawa, Y., Nishihara, T., Miyauchi, Y. \& Itami, K. Synthesis and SizeDependent Properties of [12], [16], and [24]Carbon Nanobelts. J. Am. Chem. Soc. 140, 10054-10059 (2018). 


\section{Acknowledgments}

This work was supported by the ERATO program from JST (JPMJER1302 to K.I.), the Funding Program for KAKENHI from MEXT (JP1905463 to K.I.; JP19H02701 and JP19K22183 to Y.S.), a grant-in-aid for Scientific Research on Innovative Areas from the JSPS (" $\pi$-Figuration", JP17H05149 to Y.S. and "Coordination Asymmetry", JP19H04570 to Y.H.), Toyoaki Scholarship Foundation (to Y.S.), Daiko Foundation (to Y.S.), and Asahi Glass Foundation (to Y.S.). K.W. acknowledges Special InterUniversity Researcher program in Institute for Molecular Science. The authors thank K. Yonekura, K. Takaba, S. Maki-Yonekura, N. Yasuda, RIGAKU Co., and DAICEL Co. for support with the measurements. Calculations were performed using the resources of the Research Center for Computational Science, Okazaki, Japan. ITbM is supported by the World Premier International Research Center Initiative (WPI), Japan.

\section{Author contributions}

K.I. and Y.S. conceived the concept and prepared the manuscript with feedback from the other authors. T.W., K.Y., M.K. and K.W. performed the synthetic experiments and photophysical measurements. Y.S., K.W., J.P. and Y.H. performed the computational study.

\section{Competing interests}

The authors declare no competing interests.

\section{Additional information}

Supplementary information The online version contains supplementary material available at https://doi.org/.

Correspondence and requests for materials should be addressed to Kenichiro Itami or Yasutomo Segawa. 